\title{
Conduction Intervals and Atrial Fibrillation in Chronic Kidney Disease
}

\author{
Cevher Ozcan
}

Department of Medicine, Section of Cardiology, Herat and Vascular Center, University of Chicago, Chicago, IL, USA

Atrial fibrillation (AF) is the most common sustained arrhythmia in clinical practice with increasing incidence and prevalence in the USA and around the world [1]. AF is associated with a higher risk of all-cause mortality and morbidity including hospitalizations, stroke, systemic thromboembolism, myocardial infarction, heart failure, dementia, and chronic kidney disease (CKD). An association between AF and CKD has been reported in multiple studies by the way of bidirectional relations [2, 3]. $\mathrm{AF}$ is very common in CKD patients and has significant impact on overall clinical outcomes. Yet the predictors of hospitalization with AF in patients with CKD are not well-characterized.

In this issue of American Journal of Nephrology, Scovner et al. [4] investigated whether the cardiac conduction intervals (QTc, QRS, and PR intervals) can predict hospitalization with $\mathrm{AF}$ in patients with nondialysis dependent CKD. Electrocardiograms were analyzed in 2,759 participants of the Chronic Renal Insufficiency Cohort Study, a large prospective multicenter study of patients who did not have AF at the time of enrolment. During a mean follow-up of 6.6 years, 50 hospitalizations with AF occurred among 224 participants. Shorter $(<407 \mathrm{~ms})$ or longer $(>431 \mathrm{~ms})$ baseline QTc interval was associated with higher adjusted risk of AF hospitalization. Each 10 $\mathrm{ms}$ increase in QTc from $420 \mathrm{~ms}$ was associated with a
$14 \%$ increased risk of hospitalization with AF. Longer QRS duration was an indicator for AF hospitalization in patients with a history of heart failure, while there was no significant association in those without HF. However, authors found no relation between PR interval and AF hospitalization in CKD.

This interesting study has many strengths as well as few weaknesses, as acknowledged by its authors. It provides significant data from a large and ethnically diverse population. Women are well represented in this secondary analysis of the Chronic Renal Insufficiency Cohort Study. AF hospitalization and conduction intervals are likely influenced by multiple confounding systemic processes, comorbidities, electrolytes, and variable use of medications in this population along with CKD. Event rate is relatively low and therefore the utility of QRS duration and QTc interval to serve as a risk marker for future AF hospitalization in CKD needs to be tested in a larger cohort with more effective rhythm and conduction interval surveillance. Prolonged QRS and QTc intervals characterize delay in ventricular depolarization and repolarization processes. Both have been associated with increased risk of $\mathrm{AF}$ in different populations. However, the impact of QRS and QTc interval on atrial electrical properties is not clearly explained. Scovner et al. [4] found that $\mathrm{PR}$ interval is not a predictor for AF hospitalization even 
though several studies showed prolonged PR interval is associated with AF in the general population and various disease conditions including CKD [5]. Differences between these 2 findings would benefit from further analysis. $\mathrm{AF}$ and $\mathrm{CKD}$ are associated with mutual risk factors that may have determined the clinical outcome including hospitalization and cardiac conduction intervals.

Pathogenesis of AF is a complex process with multiple contributing molecular and clinical factors including $\mathrm{CKD}$. The underlying mechanisms of the initiation and progression of AF in CKD are not yet known. Clinical and experimental studies demonstrated that arrhythmogenic atrial electro-anatomical remodeling is a critical substrate for the development of AF in patients and animal models of the disease $[1,6]$. Atrial remodeling is associated with enlarged atria, patchy fibrosis, loss of atrial muscle mass, inflammation, extracellular matrix remodeling, heterogeneous conduction velocity, and altered membrane potential and atrial refractory periods [6]. These pathogenic changes were reflected in the electrocardiogram as altered p-wave morphology and PR interval. The epidemiologic association between prolonged atrial conduction intervals and AF has been documented [5]. Prolonged conduction intervals are surrogates for pathologic structural and electrical remodeling with fibrosis and dysfunctional atrial or ventricular myocardium. Delayed conduction intervals (QRS, QTc, or PR) promote reentry and susceptibility to ectopic triggers. Conduction velocities become abnormal in fibrotic tissue with both loss of side-to-side and the longitudinal end-to-end cardiomyocyte connections [7]. This leads to micro-reentry and arrhythmias. CKD may facilitate atrial enlargement and scar resulting in conduction delay through increased atrial pressure and strain. In addition, CKD may promote myocardial fibrosis through inflammation or stress that contributes to structural remodeling of atria forming a substrate for AF. The global metabolic dysfunction in CKD may lead to electrical and mechanical instability in the atria through oxidative and metabolic stress, impaired ionic homeostasis, disrupted membrane potentials, reduced conduction velocity, and cardiomyocyte injury. This can be associated with hemodynamic change in LA with an increased pressure and volume as well as atrial refractoriness that facilitates AF. Thus, the mechanisms of the association between atrial and ventricular conduction properties need to be investigated further in CKD.

In conclusion, both $\mathrm{AF}$ and $\mathrm{CKD}$ are serious public health and economic burdens. Understanding the complex interrelations between $\mathrm{AF}$ and CKD is the first step to reduce this burden. Scovner et al. [4] provide evidence that monitoring cardiac conduction intervals, particularly QTc and QRS duration, are important in management of patients with CKD in an effort to reduce AF hospitalization. Clinicians and investigators need to address many important questions in future studies including how to prevent the coexistence of $\mathrm{AF}$ and $\mathrm{CKD}$. In addition to risk prediction via machine learning by using conduction intervals, further mechanistic and therapeutic studies are required to identify potential targets for the intervention and effective biomarkers for their association.

\section{Conflict of Interest Statement}

The author has no conflicts of interest, financial or otherwise, relevant to this manuscript.

\section{References}

1 January CT, Wann LS, Alpert JS, Calkins H, Cigarroa JE, Cleveland JC Jr, et al. 2014 AHA/ ACC/HRS guideline for the management of patients with atrial fibrillation: a report of the American College of Cardiology/American Heart Association Task Force on Practice Guidelines and the Heart Rhythm Society. J Am Coll Cardiol. 2014;64(21):e1-76.

2 Watanabe H, Watanabe T, Sasaki S, Nagai K, Roden DM, Aizawa Y. Close bidirectional relationship between chronic kidney disease and atrial fibrillation: the Niigata preventive medicine study. Am Heart J. 2009;158(4): 629-36.
3 Bansal N, Fan D, Hsu CY, Ordonez JD, Marcus GM, Go AS. Incident atrial fibrillation and risk of end-stage renal disease in adults with chronic kidney disease. Circulation. 2013;127(5):569-74.

4 Scovner KM, Correa S, Claggett BL, Barrett CD, Waikar SS, Solomon S, et al. Specific electrocardiograph intervals predict hospitalization with atrial fibrillation in those with chronic kidney disease. Am J Nephrol. 2021.
5 Cheng S, Keyes MJ, Larson MG, McCabe EL, Newton-Cheh C, Levy D, et al. Long-term outcomes in individuals with prolonged PR interval or first-degree atrioventricular block. JAMA. 2009;301(24):2571-7.

6 Ozcan C, Battaglia E, Young R, Suzuki G. LKB1 knockout mouse develops spontaneous atrial fibrillation and provides mechanistic insights into human disease Process. J Am Heart Assoc. 2015;4(3):e001733.

7 de Bakker JM, van Capelle FJ, Janse MJ, Tasseron S, Vermeulen JT, de Jonge N, et al. Slow conduction in the infarcted human heart. "Zigzag" course of activation. Circulation. 1993;88:915-26. 